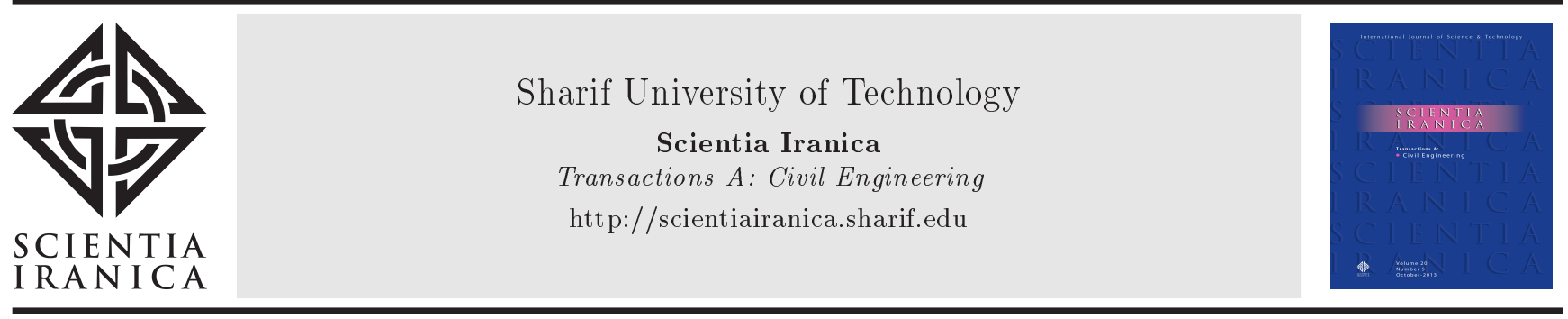

Invited Paper

\title{
An efficient method for reliability estimation using the combination of asymptotic sampling and weighted simulation
}

\author{
A. Kaveh* and A. Dadras Eslamlou \\ School of Civil Engineering, Iran University of Science and Technology, Narmak, Tehran, Iran.
}

Received 23 January 2019; accepted 8 April 2019

\section{KEYWORDS}

Reliability index;

Failure probability;

Sampling method;

Asymptotic behavior;

Weighted simulation.

\begin{abstract}
In this paper, an efficient reliability method is proposed. Asymptotic Sampling (AS) and Weighted Simulation (WS) are two basic tools of the presented method. In AS, the standard deviation of the distributions is amplified at several levels to find an adequate number of failed samples; then, by using a simple regression technique, the reliability index is determined. The WS is another method that uses the uniform distribution for sampling, in which the information about the distributions of the variables is taken into account through the weight indexes. The WS provides interesting flexibility where a sample generated for a specific standard deviation can be used as a sample for another standard deviation without having to reevaluate the limit state function. In AS, the deviations of variables are scaled in each step, where one can use the flexibility of the WS to decrease the required calls of limit state function. Using this technique results in a new efficient method, so-called Asymptotic Weighted Simulation (AWS). In addition, using the strengths of both AS and WS can be considered another superiority of the hybrid version. Performance of the presented method is investigated by solving several mathematical and engineering examples.

(C) 2019 Sharif University of Technology. All rights reserved.
\end{abstract}

\section{Introduction}

Failures in industrial systems may result in catastrophic consequences. In order to design safe systems and prevent severe consequences, the engineers have to deal with the arising uncertainties $[1,2]$. The uncertainty comprises different types, including physical, statistical, and model uncertainties [3]. For instance, some variations may occur in the forces acting on the

*. Corresponding author. Tel.: +982144202710; Fax: +9821 ry240398

E-mail addresses: alikaveh@iust.ac.ir (A. Kaveh); armin_dadras@alumni.iust.ac.ir (A. Dadras Eslamlou)

doi: $10.24200 /$ sci. 2019.21367 structure during its lifetime. These variabilities are usually quantified by the probabilistic distributions. Accordingly, the safety or failure state of the structures can be represented by the probabilistic models. Structural safety requires the probability of failure to become extremely small [4]. The reliability methods and their applications have attracted a great deal of interest due to their important role in system safety $[5,6]$.

The failure probability of the systems can be stated in the form of the following multiple integral:

$$
\begin{aligned}
P_{f} & =\operatorname{Prob}\left[g\left(X_{1}, X_{2}, \cdots, X_{n}\right) \leq 0\right] \\
& =\int_{g(X) \leq 0} \cdots \int f_{X}(X) d X,
\end{aligned}
$$


where $X_{i}(i=1,2, \cdots, n)$ is the $i$ th basic random variable representing load, structural dimensions, or other existing uncertain quantities. The functions $g(X)$ and $f(X)$ are the limit state function and the joint Probability Density Function (PDF) of $X$, respectively. The negative values for the limit state function represent the failure set [7]. The reliability index $(\beta)$ is defined as:

$$
\beta=\phi^{-1}\left(1-P_{f}\right) \text {. }
$$

In this equation, $\phi^{-1}($.$) is the inverse standardized$ Gaussian distribution function. When the number of random variables increases or the failure regions have complicated shapes, evaluation of Eq. (1) becomes extremely difficult [7]. Therefore, various numerical and computational approximate methods are introduced by researchers as outlined in the following.

Hasofer and Lind, in their groundbreaking article [8], by definition of the reliability problem in standard Gaussian space, established the First-Order Reliability Method (FORM). In this paper, by using Rosenblatt-transformation, the correlated nonGaussian variables $X$ were transformed to uncorrelated Gaussian variables $U$ with zero mean and unit variance. For simplifying the integration, a linear approximation (the first-order Taylor expansion) was utilized. By maximizing the Probability Density Function (PDF) within the failure domain, $u^{*}$ was found through an optimization process. The point $X^{*}$ corresponding to $U^{*}$ was called the design point. Finally, the reliability index $(\hat{a})$ was calculated. Further details about FORM are available in [7].

The mathematical definition of failure probability given in Eq. (1) can be rewritten in the following form:

$$
\begin{gathered}
P_{f}=\int_{-\infty}^{+\infty} \int_{-\infty}^{+\infty} \cdots \int_{-\infty}^{+\infty} I_{g}\left(X_{1}, \cdots, X_{n}\right) f_{X_{1}, \cdots, X_{n}} \\
\left(X_{1}, \cdots, X_{n}\right) d X_{1}, \cdots, d X_{n} .
\end{gathered}
$$

Herein, if $g\left(X_{1}, \cdots, X_{n}\right) \leq 0$, then $I_{g}\left(X_{1}, \cdots, X_{n}\right)=$ 1 ; otherwise, $I_{g}\left(X_{1}, \cdots, X_{n}\right)=0$.

Considering a large collection of samples $(m)$, the failure probability can be estimated as [9]:

$$
P_{f}=\frac{1}{m} \sum_{k=1}^{m} I_{g}\left(X^{(k)}\right) .
$$

As seen, the new suggested form for calculating the failure probability, known as the Monte-Carlo Simulation (MCS), is not involved with the aforementioned difficulties of integration. However, it should be noted that for achieving accurate estimations, especially for small values of $P_{f}$, the number of samples must be sufficiently large. For the sake of computational cost saving, various strategies have been proposed that artificially produce more samples than the primary distribution functions. For instance, the Importance Sampling method was suggested, in which the failure probability was estimated using the following formulation:

$$
P_{f}=\frac{1}{m} \sum_{k=1}^{m} \frac{f_{X}(X)}{h_{Y}(X)} I_{g}\left(X^{(k)}\right),
$$

where $h_{Y}(X)$ is a positive weighting function. An ideal weighting function requires a foreknowledge of failure probability, while this probability is usually unknown. Bucher proposed a fast procedure which utilized results from the simulation for adapting $h_{Y}(X)$ for the specific problem [10].

In 2009, Bucher also developed an efficient MC based simulation method, so-called Asymptotic Sampling (AS) [11]. This method provided an accurate estimation of the failure probability with perceptibly low computational cost. In asymptotic sampling, by producing samples with scaled distributions in several levels and fitting a regression, the reliability index is calculated through an extrapolation. On the other hand, in 2012, Rashki et al. introduced an approximate method in which, by generating a small number of uniformly distributed samples and multiplying the probability density as a weighted index at each sample, the failure probability was calculated [12]. Since this Weighted Simulation (WS) method utilized the uniform distribution, it was independent of the standard deviation of the variables and failure probability was taken into account by weight indexes. This is a substantial point which means that the samples produced for a specific standard deviation can be utilized for another sampling with different deviation, which is desired in AS. This paper presents a hybrid framework for incorporating the advantages of WS in the AS to decrease the number of sample evaluations as well as for entering the advantages of AS into the WS, thereby achieving an efficient method.

In recent years, many techniques have been developed for optimal design of structures and systems $[13,14]$. These methods usually require a significant number of analyses $[15,16]$. Especially when the Reliability-Based Design Optimization (RBDO) is considered, the amount of computations may grow greatly $[17,18]$. The huge amount of computations may dissuade engineers to design reliable systems due to limitations on computing resources. Therefore, proposing such efficient reliability and sampling methods would make an important contribution to this field.

The rest of this paper is organized as follows. In Section 2, the AS and WS methods are briefly reviewed. After providing the necessary background, a new hybrid method is developed in Section 3. In Section 4, different numerical experiments are chosen 
to examine the efficiency of the new method. Finally, the conclusions are drawn in Section 5.

\section{Background theory}

The proposed method is based on Asymptotic Sampling (AS) and Weighted Simulation (WS) methods, which are described in the following subsections.

\subsection{Asymptotic sampling}

As mentioned before, an important strategy to increase the efficiency of reliability sampling is to produce more samples in the failure domain. AS attempts to increase the chance of dropping samples in the failure domain by increasing the standard deviations. However, it should be noted that some increment of the deviations may result in invalidity of the calculated failure probability. In AS, this issue is resolved through a certain asymptotic behavior of the failure probability in the independent and identically distributed (i.i.d.) Gaussian space $[11,19]$. Using the asymptotic behavior also results in reliability assessment with fewer samples. Assuming a linear limit state function as:

$$
g(X)=\sum_{k=1}^{N} a_{k} X_{k}
$$

the variance of $Y=g(x)$ will be:

$$
\sigma_{y}^{2}=\sum_{k=1}^{N} a_{k}^{2}
$$

If the standard deviations of all variables are scaled to $\frac{1}{f}$, the variance of $Y$ will be changed to:

$$
\sigma_{y}^{2}=\frac{1}{f^{2}} \sum_{k=1}^{N} a_{k}^{2}
$$

thus, the distribution function, $F_{Y}(\xi)$, becomes:

$$
F_{Y}(\xi)=\phi\left(\frac{\xi f}{\sigma_{y}}\right)=\phi\left(\frac{\xi f}{\sqrt{\sum_{k=1}^{N} a_{k}^{2}}}\right),
$$

and the safety index for an arbitrary standard deviation $(\sigma)$ can be stated as follows:

$$
\beta(f)=\frac{\xi f}{\sqrt{\sum_{k=1}^{N} a_{k}^{2}}} .
$$

According to Eq. (10), the following ratio is independent of the value of $f[20]$ :

$$
\frac{\beta(f)}{f}=\text { const. }
$$

As proven in [19], Eq. (11) for nonlinear cases is true only when $f$ approaches infinity, as shown in the following equation:

$$
\lim _{f \rightarrow \infty} \frac{\beta(f)}{f}=\text { const. }
$$

This asymptotic property of the safety index is employed to construct a regression model as:

$$
\frac{\beta(f)}{f}=A+\frac{B}{f^{2}},
$$

where the coefficients $A$ and $B$ can easily be determined by a regression analysis based on samplings with different deviations. This formulation ensures the convergence of $\frac{\beta(f)}{f}$ to a constant value $(A)$.

The pseudo-code of AS is provided in the following.

\section{Procedure AS}

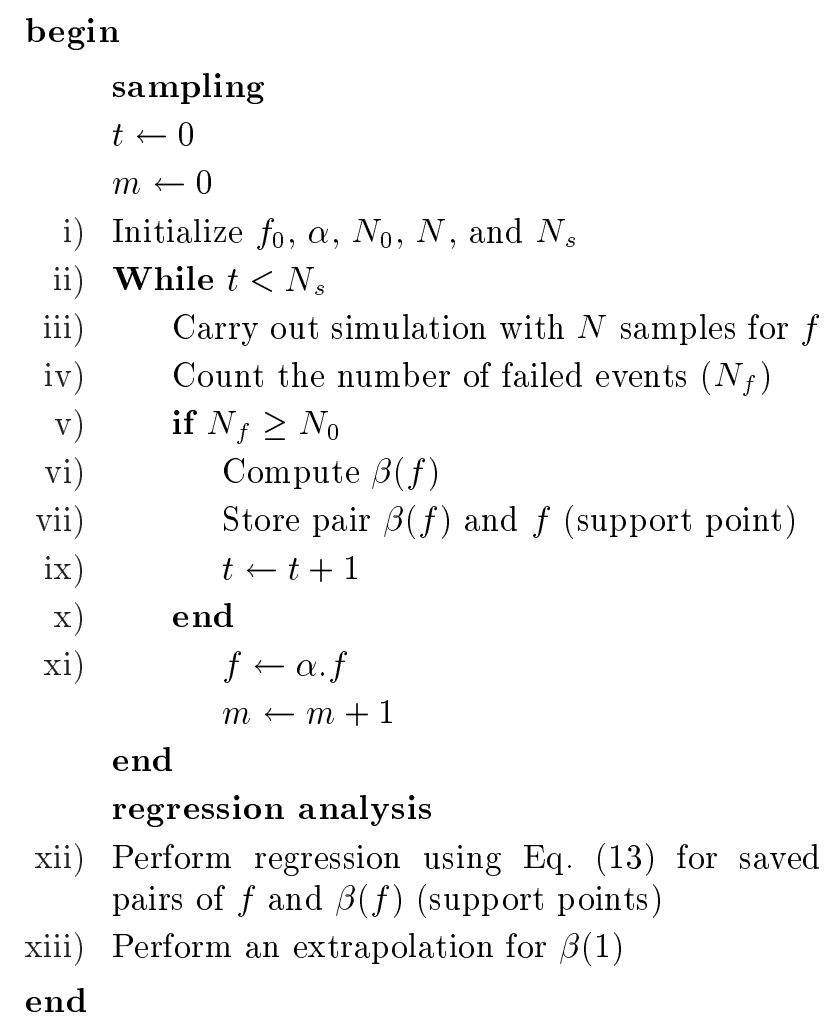

The pseudo-code of AS is clarified in the following:

i) In the sampling step, the values of $f_{0}$ (the initial value of $f$ ), $\alpha$ (the decreasing factor of $f$ ), $N_{0}$ (the lowest value of occurring failures), and $N_{s}$ (the number of support points for regression) are determined. Here, $f_{0}$ is set to 1 and $\alpha$ is considered as 0.9 ;

ii) To get enough support points, the loop is repeated until the number of $N_{s}$ pairs are stored. Here, each repetition of the loop is called a "level";

iii) MCS is performed by considering the scaling factor $f$; 


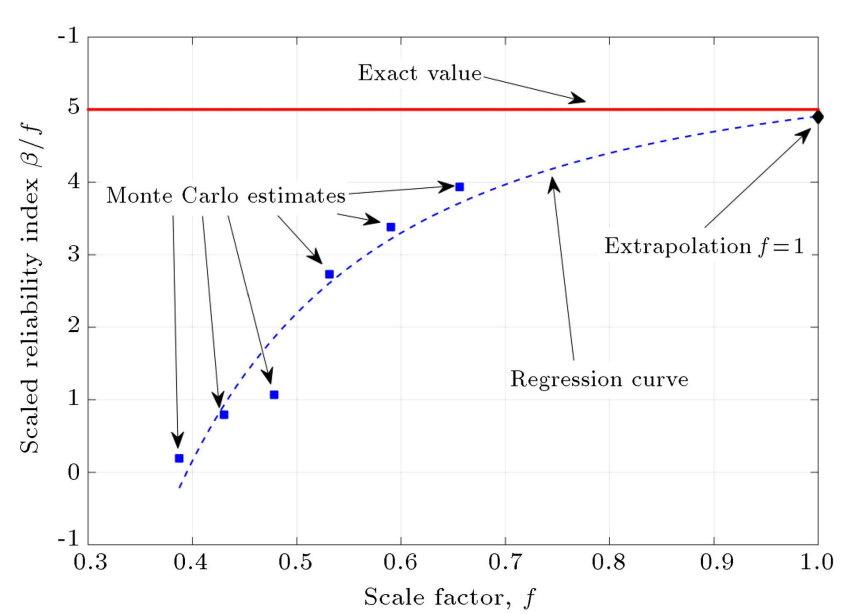

Figure 1. Regression concepts of the asymptotic sampling.

iv) The number of failed samples is counted to be compared with $N_{0}$

$\mathrm{v}$ to ix) The number of failed samples $\left(N_{f}\right)$ is compared with $N_{0}$; if the condition is fulfilled, then the reliability index is calculated and the pair $(f, \beta)$ is stored;

$\mathrm{x}, \mathrm{xi})$ The factor $f$ is decreased to $\alpha . f$;

xii) A regression model is constructed according to the stored support points and Eq. (13) (Figure 1). Thus, the coefficients $A$ and $B$ are determined;

xiii) The reliability index of the model $(\beta(1))$ is calculated. As seen in Eq. (13), the reliability index is equal to $A+B$.

It should be noted that in $\mathrm{AS}$, the number of evolutions of the limit state function is equal to $\times$ final value of $m$, which is equal to or higher than $N \times N_{s}$. An illustration of this method is provided in Figure 2 .

\subsection{Weighted simulation method}

In WS, after the generation of the samples using the uniform random distribution and evaluation of the limit state function, the probability of failure is approximated by:

$$
P_{f}=\frac{1}{m} \cdot \frac{\sum_{k=1}^{m} I_{g}\left(x^{(k)}\right) \cdot W_{k}}{\sum_{k=1}^{m} W_{k}},
$$

where the weight indexes $\left(W_{k}\right)$ are calculated via the following relationship:

$$
W_{k}=\prod_{j=1}^{s} f_{j}\left(x_{j}^{(k)}\right),
$$

in which $s$ is the number of random variables and $f_{j}$ is the PDF of the $j$ th variable.

The pseudo-code of the WS method is provided in the following:

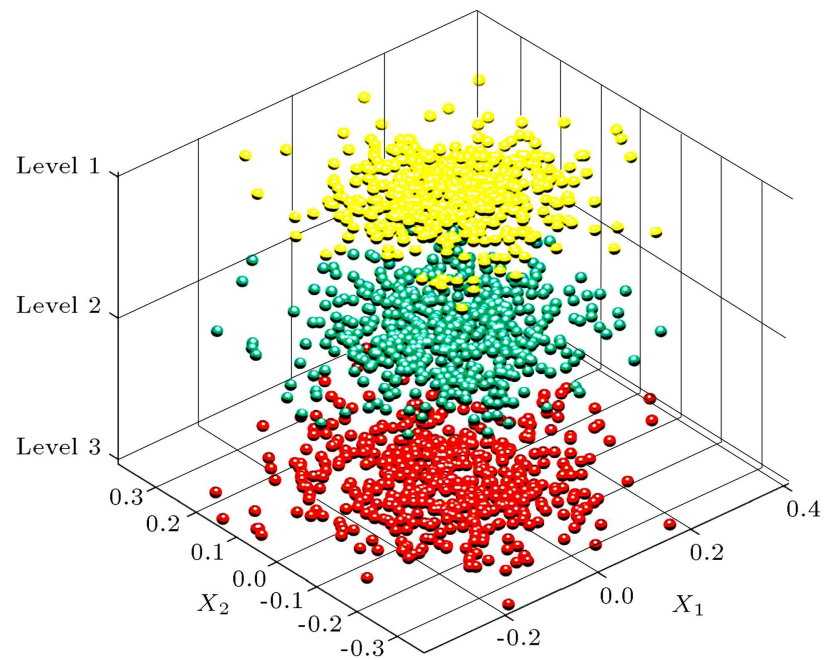

Figure 2. Illustration of the asymptotic sampling for three levels.

\section{Procedure WS}

\section{begin}

i) Select proper intervals;

ii) Distribute the uniform sample in the selected interval;

iii) Calculate the weight indexes;

iv) Evaluate the limit state function;

v) Approximate the failure probability. end

The following explanations further clarify the steps of the pseudo-code:

i) In order to find the region of sampling, the intervals must be determined. Two techniques have been suggested for this purpose [12]. The first technique determines the maximum and minimum values of samples using the corresponding sample boundaries of an MCS with the PDF of the variables;

ii) The samples are distributed uniformly within the intervals calculated in the previous step;

iii) The weight index of each sample is calculated by Eq. (15);

iv) The limit state for each of the samples is evaluated and $I_{g}$ is calculated;

$\mathrm{v}$ The failure probability is approximated using Eq. (14).

\section{An efficient asymptotic weighted sampling method}

As mentioned in Section 2.2, the WS method utilizes the uniform distribution instead of other probabilistic distributions. This feature provides flexibility for WS 
and, by modifying the weight indexes, the generated samples can be adapted for different distributions, instead of generating new samples and re-evaluation of the limit state function. Sometimes the limit state functions are complex and the evaluation of samples is computationally expensive. From this point of view, the flexibility feature of WS is attractive. On the other hand, as mentioned in Section 2.1, AS has a loop in which several samplings with different scaled standard deviations are generated and evaluated. Employing the flexibility feature of WS makes it possible to use the samples generated in the previous levels for the next levels of the loop. Indeed, the AWS utilizes the WS for sampling and making support points of AS, where applying the flexibility feature decreases the number of generated samples in the next levels. The steps of this new hybrid method, known as the Asymptotic Weighted Sampling (AWS), are designed and provided in the following:

\section{Procedure AWS}

begin

\section{Initialization}

$t \leftarrow 0$

$m \leftarrow 0$

i) Initialize $f_{0}, \alpha, N_{0}, N$, and $N_{s}$

\section{Sampling}

ii) While $t<N_{s}$

iii) Select the proper intervals for sampling like WS;

iv) Calculate the number of samples required to generate out of the previous intervals $\left(N_{r e q}\right)$;

v) Remove the number of $N_{\text {req }}$ samples;

vi) Generate the number of $N_{r e q}$ samples out of the previous intervals using uniform random distribution;

vii) Evaluate only the newly generated samples;

viii) Count the number of failed events $\left(N_{f}\right)$;

ix) $\quad$ if $N_{f} \geq N_{0}$

$\mathrm{x}) \quad$ Calculate the weight indexes using Eq. (15);

xi) Estimate the failure probability by Eq. (14) and compute $\beta(f)$;

xii) $\quad$ Store pair $\beta(f)$ and $f$;

xiii) $\quad t \leftarrow t+1$

$$
\text { end }
$$

xiv) $\quad f \leftarrow \alpha \cdot f$

$$
\text { end } m \leftarrow m+1
$$

regression analysis

xv) Perform regression using Eq. (13) for saved pairs of $f$ and $\beta(f)$ xvi) Perform an extrapolation for $\beta(1)$

end

The steps of AS-WS are discussed here. For the sake of brevity, the similar steps refer to the previous section.

i) In the first step, the values of $f_{0}, \alpha, N_{0}, N$, and $N_{s}$ are initialized as explained in Section 2.1;

ii, iii) To obtain the number of $N_{s}$ support points, a loop is repeated. At the first step of the loop, the proper intervals must be determined as explained in Section 2.2;

iv) Since the samples are distributed uniformly, the required number of new samples in each step is defined by Eq. (16):

$$
N_{r e q}^{m}=N \times\left(1-\frac{V_{m-1}}{V_{m}}\right),
$$

where $V_{m}$ is the volume of the $m$ th sampling space, defined by:

$$
V_{m}=\prod_{j=1}^{s} L_{j}^{m},
$$

where $V$ is determined by multiplying the length of all intervals $\left(L_{j}^{m}\right)$ described in step iii. Since at the first level of the loop, there is no generated sample to be used, $N_{r e q}^{0}$ is assumed to be equal to $N$. Because of growing of the deviations, it is expected that the bounds of the new intervals will be further than those of the previous levels. However, in some levels, it is possible that the bounds of the new intervals may be trapped in the previous one. In this cases, the trapped bound is replaced by the corresponding bound of the previous interval. Hence, it is expected that the value of $\left(\frac{V_{m-1}}{V_{m}}\right)$ should be equal to or less than one. According to Eq. (16), $N_{r e q}^{m}$ will be equal to or less than $N$, which is shown in Figure 3. Comparing Figure 3 with Figure 2, it is clear that, unlike AS, the proposed method avoids making new samples in intervals of the previous levels (void space). The numbers of generated samples are also lower than $N$, while the AS generates the number of $N$ samples in all levels;

v) In order to have the number of $N$ samples, the same number of generated new samples $\left(N_{\text {req }}\right)$ should be removed from the samples. Here, two types of removing are suggested; in the first type (AWS-1), the samples are selected randomly, while in the second type (AWS-2), some of the failed samples are not removed. In AWS-1, the number of failed 


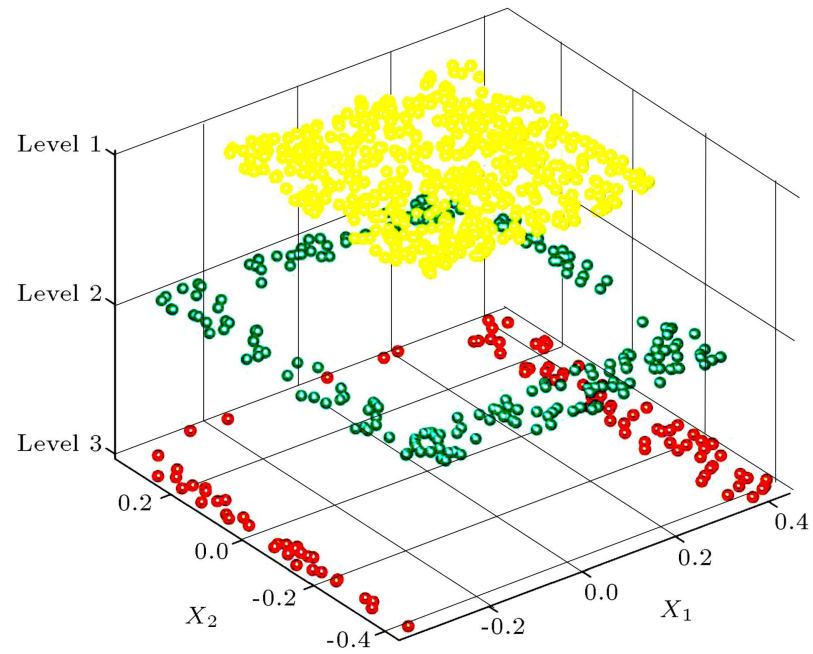

Figure 3. The schematics of sampling by the proposed method for a $2 \mathrm{D}$ problem in three levels.

samples which should be saved is equal to $\min \left(N_{0}, N f\right)$. Once the condition of $\left(N_{f} \geq\right.$ $N_{0}$ ) is satisfied, satisfaction of the next levels is guaranteed. In Section 4, both of the proposed versions are investigated;

vi, vii) Only the newly generated samples are eval- uated and the number of failed samples is counted.

Other steps are identical to the steps of the AS and WS methods provided in Section 2. Figure 4 displays the flowchart of the proposed method.

\section{Numerical experiments}

To examine the performance of the proposed method, several problems are solved and the results are discussed and compared with other methods. All the algorithms and problems were implemented in MAT$\mathrm{LAB}$, and the tests were accomplished on a $2.40 \mathrm{GHz}$ Intel Core(TM) with 8.00 GB-RAM laptop. In order to provide statistical confidence, each problem was run 30 times independently. It should be noted that while for the MCS and WS algorithms, we can exactly set the number of state function evaluations, it cannot be precisely controlled in the AS, AWS-1 , and AWS-2 methods. Therefore, we adjusted the average amounts of the Number of Function Evaluations $(\overline{N F E})$ to make them close to the aforementioned numbers. During computations, we managed to ensure that the values of $\overline{N F E}$ for the proposed methods (AWS-1 and AWS2) were smaller than those for other algorithms. Par-

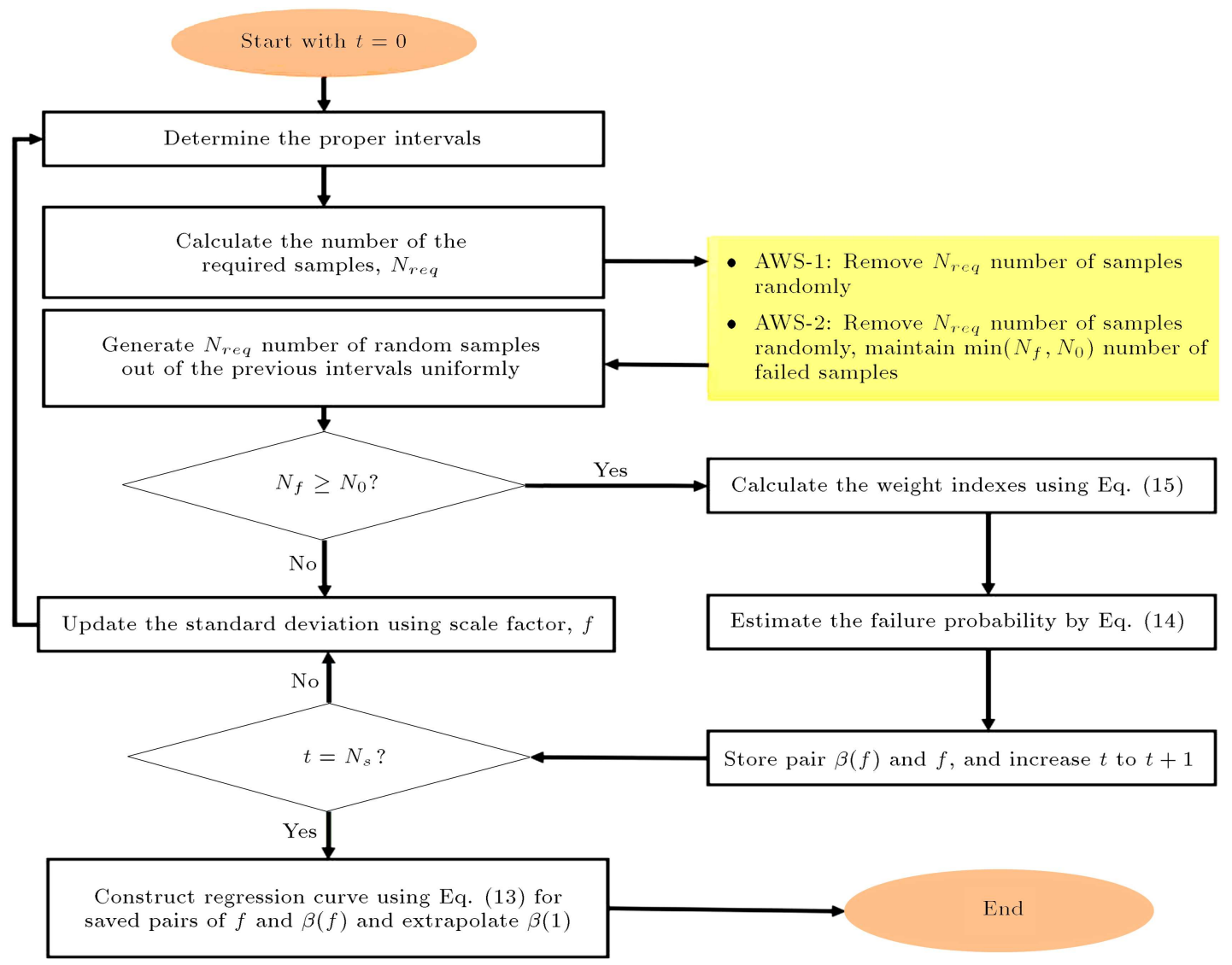

Figure 4. The flowchart of the introduced AWS method. 
ticularly, in parameter analysis, $N$ (not $\overline{N F E}$ ) for the proposed methods was equalized to that for the AS.

\subsection{Example 1: A mathematical example with a small failure probability}

The first example is a challenging mathematical example with a small failure probability $P_{f}=1.41 \times 10^{-13}$ and high reliability index $\beta=7.30$. Suppose $X$ is a two-dimensional variable $X=\left(X_{1}, X_{2}\right)$, where both $X_{1}$ and $X_{2}$ are independent random variables which follow a normal standard distribution with zero mean and unit standard deviation. The failure event is defined as $\min \left\{x-x_{r 2}, x-x_{l 2}\right\}<1$, so the limit state function can be defined as $g(x)=\min \left\{x-x_{r}, x-x_{l}\right\}-1$, where $x_{r}=(8,2)$ and $x_{l}=(-8,2)$ [21].

For the sake of fairness in comparison, two sets of results with approximately equal $\overline{N F E} s$ are represented in Table 1. Considering the average value of $N F E(\overline{N F E})$ equal to $3500, N_{0}=1$ and $N s=6$, neither MCS nor WS can find any failed sample. In this set, the values of the averages of reliability indexes $(\bar{\beta})$ for both versions of AWS are meaningfully close to the exact value (7.30). The calculated values of Root Mean Square Error $(R M S E)$ for the AWS-1 and 2 are considerably lower than those for AS, which shows the good accuracy of this method in comparison with AS. The percentages of the Coefficient of Variation $(\mathrm{CV})$ for AWS-1 and 2 are $5.07 \%$ and $19.78 \%$, respectively, while the AS method has $118.73 \%$, which shows slight change in the results of the proposed methods. The results of the AS, AWS-1, and AWS-2 are illustrated in Figure 5. As it can be seen, the reliability indexes calculated by AWS- 1 and 2 oscillate in a narrow range, while those of AS oscillate in a wider unstable range. Again, in the second set, MCS and AS cannot find any failed sample. In this set, the average values of the reliability indexes calculated by AWS- 1 and 2 match the exact answer (7.30). Also, the $C V$ and $R M S E$ for both versions of the proposed methods are less than those for the AS method.

To monitor the samples of AWS method against WS and AS methods, the positions of samples are

Table 1. Comparison of the performances of the different methods for Example 1.

\begin{tabular}{cccccc}
\hline & MCS & AS & WS & AWS-1 & AWS-2 \\
\hline$\overline{N F E}$ & 3500 & 3700 & 3500 & 3367 & 3309 \\
$\bar{\beta}$ & - & 4.76 & - & 7.51 & 7.31 \\
$R M S E$ & - & 6.11 & - & 0.42 & 1.42 \\
$C V(\%)$ & - & 118.73 & - & 5.07 & 19.78 \\
\hline$\overline{N F E}$ & 35000 & 37167 & 35000 & 26668 & 26311 \\
$\bar{\beta}$ & - & 8.73 & - & 7.30 & 7.30 \\
$R M S E$ & - & 1.79 & - & 0.45 & 0.44 \\
$C V(\%)$ & - & 15.88 & - & 3.43 & 3.34 \\
\hline
\end{tabular}

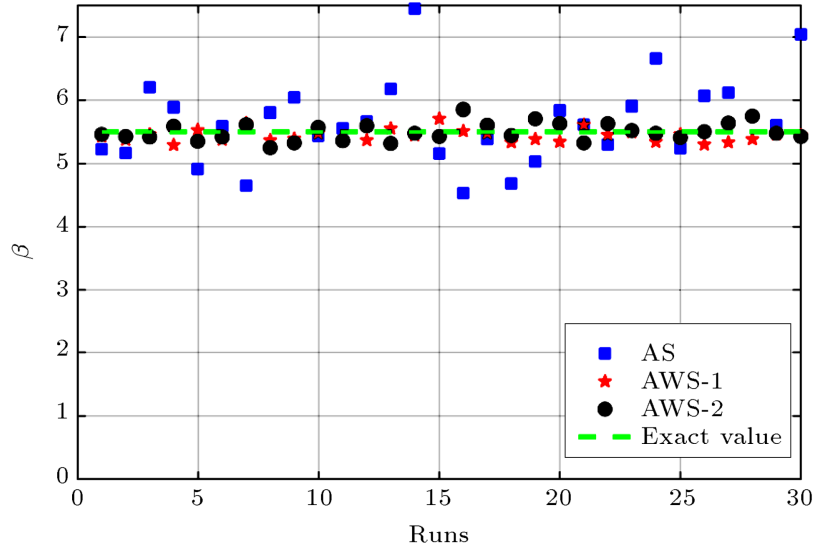

Figure 5. Reliability indexes obtained in 30 runs by different algorithms.

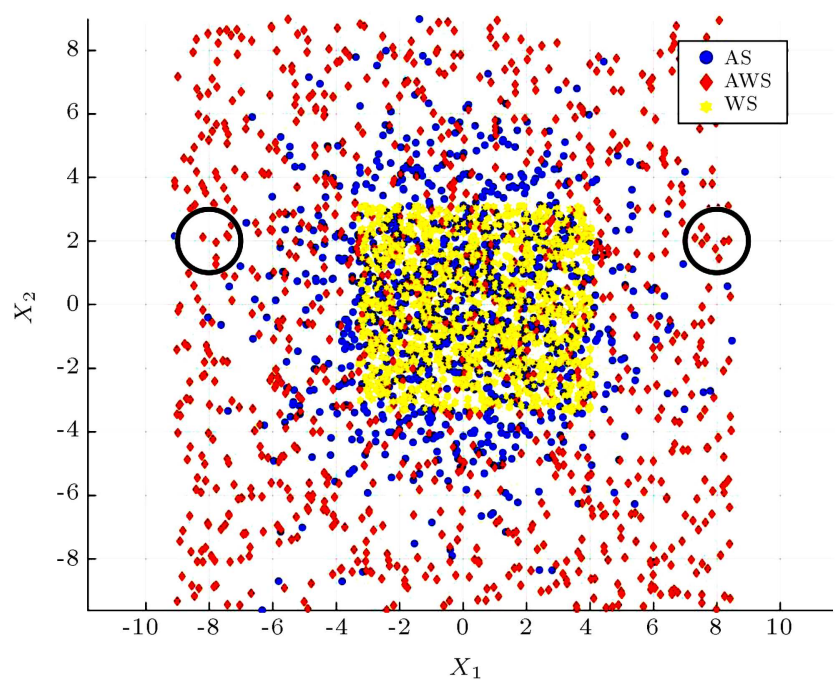

Figure 6. Positions of samples generated by different methods for Example 1.

scattered in Figure 6. As can be seen, since the standard version of WS does not have the ability to search wider spaces, none of its samples fall in the failure domain (black rings). On the other hand, in the 10th level of AS, it also cannot drop any of the samples inside the black rings, because it follows Monte-Carlo simulation, which generates the samples near the mean. On the other hand, AWS method, using the WS with intervals determined by the same Monte-Carlo samples, drop more than 15 samples inside the failure regions.

The sensitivity of $C V, R M S E$, and $\overline{N F E}$ for the AS method and both versions of AWS to $N_{s}$ and $N_{0}$ is investigated and the results are illustrated in Figure 7. According to the provided results, in most cases, the proposed algorithms, by increasing the number of support points, lead to a decrease in the values of $R M S E$ and $C V$. Considering $N_{s}=N_{0}=1$, creates interesting versions of the MCS (for AS) and WS (for AWS-1 and 2), which, after finding the first sample and creating the first support point, go to calculate 


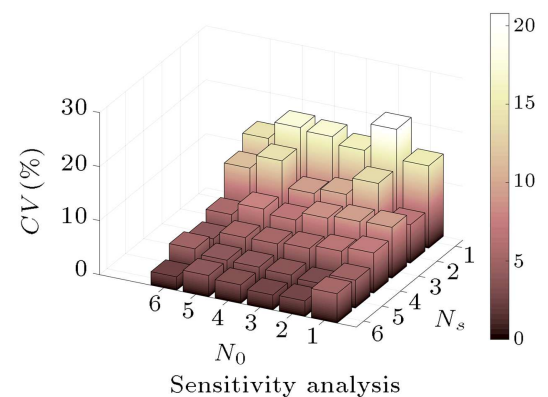

Sensitivity analysis of $C V$ for AWS-1

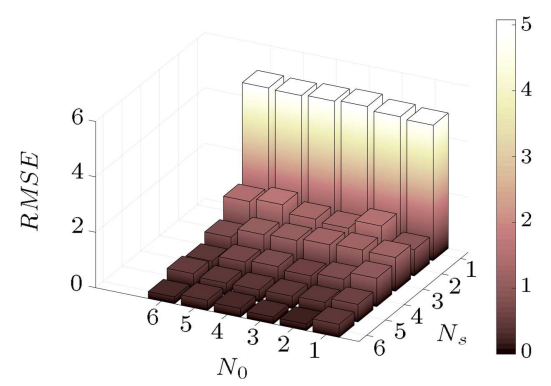

Sensitivity analysis of $R M S E$ for AWS-1

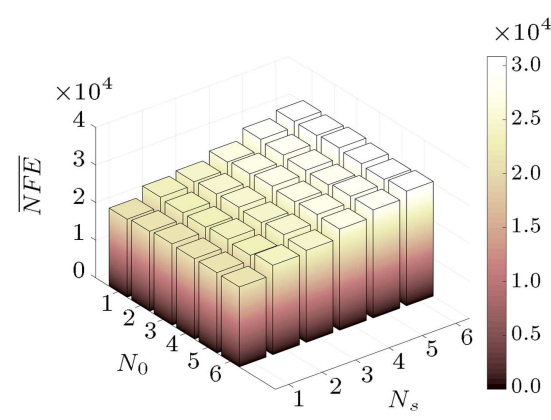

Sensitivity analysis of $\overline{N F E}$ for AWS-1

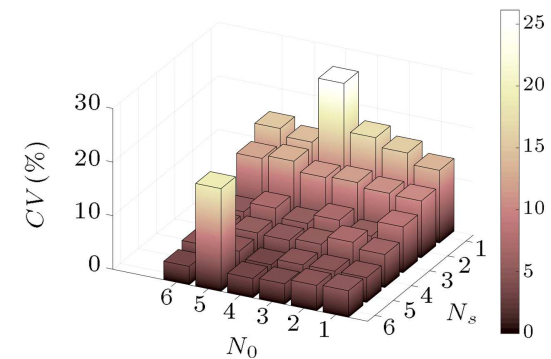

Sensitivity analysis of $C V$ for AWS-2

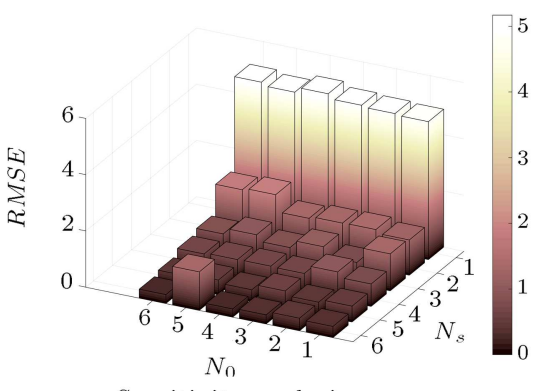

Sensitivity analysis of RMSE for AWS-2

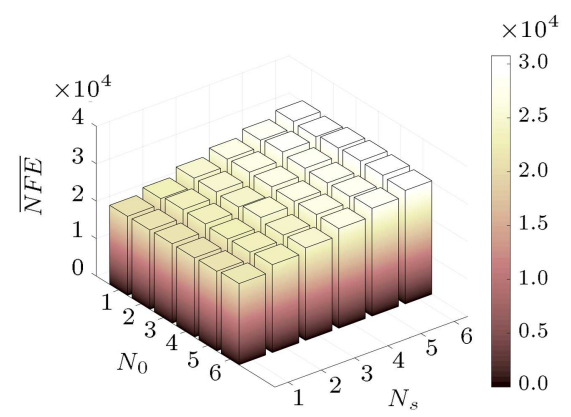

Sensitivity analysis of $\overline{N F E}$ for AWS-2

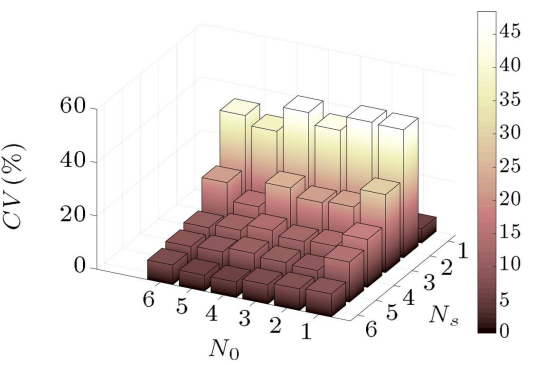

Sensitivity analysis of $C V$ for AS

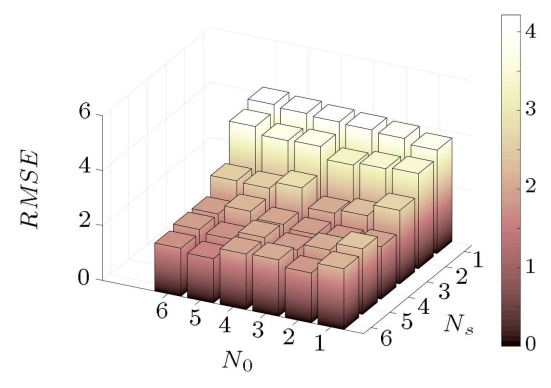

Sensitivity analysis of $R M S E$ for AS

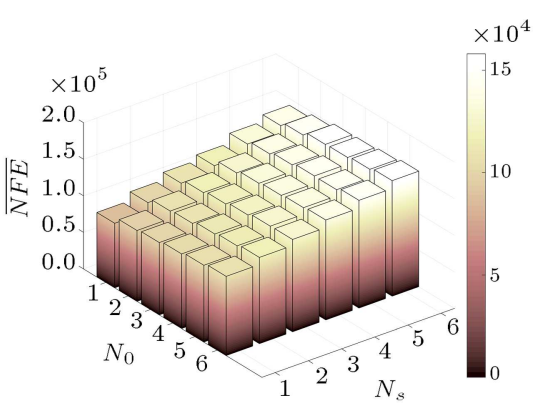

Sensitivity analysis of $\overline{N F E}$ for AS

Figure 7. Sensitivity analysis of the $C V, R M S E$, and $\overline{N F E}$ for Example 1.

the reliability index. However, considering only one support point is not compatible with regression process and, as can be seen in Figure 7, for this example, results in high values of $\mathrm{CV}$ and RMSE. Despite the single support point, $C V$ and RMSE of AWS-1 and 2 are about $7 \%$ and 0.8 , respectively, while these values for AS are about $20 \%$ and 2.4. The values given for the average number of limit state functions (for $N=10000$ ) show that AS utilizes almost 88333 to 158000 function evaluations, while the values of $\overline{N F E}$ for the proposed methods are about 21121 for a single support point and 30864 for six support points, which show four to five times reduction. It also can be seen that the values of $\overline{N F E}$ for AWS-1 and AWS-2 are almost the same.

The regression curve and support points of the algorithms are plotted in Figure 8. This figure shows not only better accuracy of the proposed methods, but

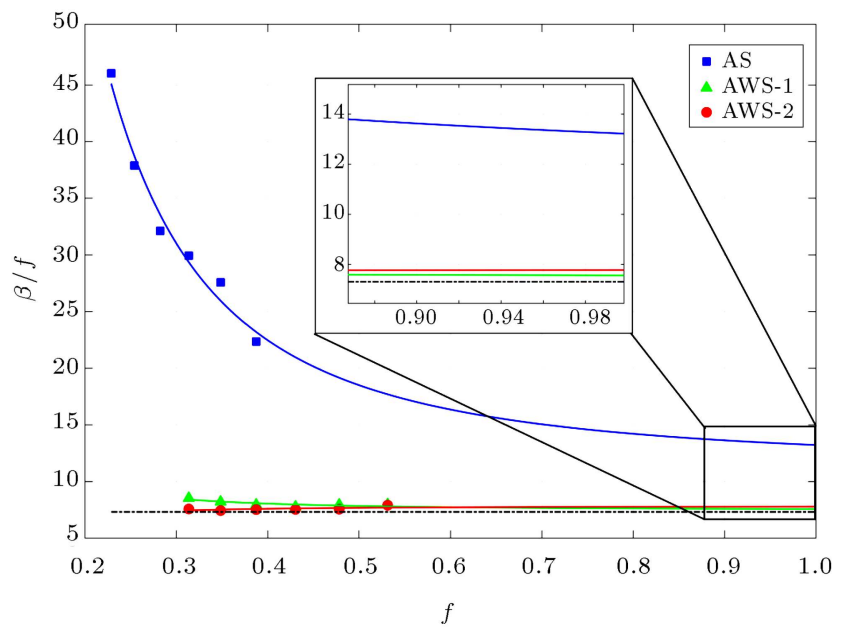

Figure 8. The regression curves of AS method $(N F E=40000)$, AWS-1 method $(N F E=30830)$, and AWS-2 method $(N F E=28013)$. 
also quick access of the hybrid methods (7th level) compared to the AS method (10th level).

\subsection{Example 2: Hypothetical limit state}

The explicit nonlinear equation given in Eq. (18) is selected as the limit state function of the second example [22]:

$$
g(X)=\exp \left(0.2 X_{1}+6.2\right)-\exp \left(0.47 X_{2}+5.0\right)
$$

where $X_{1}$ and $X_{2}$ are independent and have standard normal distributions. The failure probability is 0.00937 and the reliability index is 2.35 [22]. According to Figure 9(a), Eq. (18) has higher nonlinearity in the safe region and the limit state is linear. As it can be seen in Figure 9(b), the samples of MCS are gathered along the mean point $(0,0)$ and the distributions of the samples of WS are more uniform, but still all the samples are in the safe region (lower than the red line). The asymptotic methods (AS and AWS) find failed samples (upper than the red line); however, the failed samples of the AWS are more than those of the AS. Indeed,
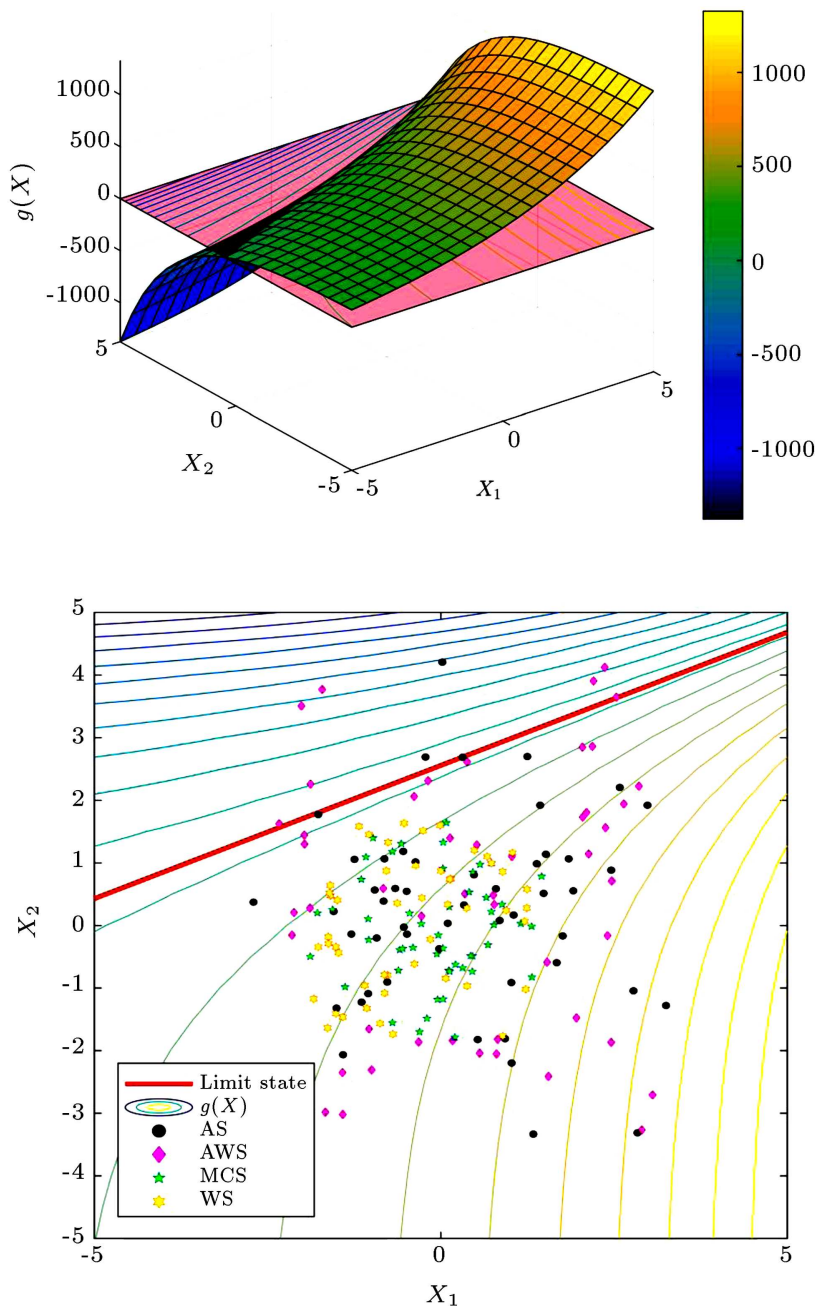

Figure 9. Schematics of Example 2: (a) Surf of limit state function, and (b) sampling by different methods.
Table 2. Comparison of the performances of the different methods for Example 2.

\begin{tabular}{cccccc}
\hline & MCS & AS & WS & AWS-1 & AWS-2 \\
\hline$\overline{N F E}$ & 215 & 215 & 215 & 208 & 205 \\
$\bar{\beta}$ & - & 2.46 & - & 2.62 & 2.58 \\
$R M S E$ & - & 0.71 & - & 0.36 & 0.32 \\
$C V(\%)$ & - & 31.92 & & 9.67 & 8.94 \\
\hline$\overline{N F E}$ & 2000 & 2353 & 2000 & 1950 & 1943 \\
$\bar{\beta}$ & 2.35 & 2.43 & 2.38 & 2.35 & 2.37 \\
$R M S E$ & 0.087 & 0.19 & 0.06 & 0.085 & 0.077 \\
$C V(\%)$ & 3.75 & 7.35 & 2.43 & 3.68 & 3.10 \\
\hline
\end{tabular}

because of the uniform distribution conducted in AWS, the possibility of the failure of its samples is higher.

Two sets of statistical results obtained by different methods are given in Table 2. As can be seen, only asymptotic methods (AWS and AS) are able to estimate the reliability index for an average number of 215 function evaluations. The estimations provided for the first set also demonstrate the lower $R M S E$ and $C V$ of the hybrid methods (AWS-1 and 2) than those of the standard AS. For the second set, the $\overline{N F E}$ is almost 2000, where, because of the high failure probability of this example, all methods reach nearly acceptable results.

The parameter studies of the asymptotic methods for $N=10000$ are illustrated in Figure 10. As it can be seen, unlike the AS, $C V$ values for most cases of the introduced methods are less than one percent. The average $R M S E$ of the AS is about 0.04, while those of the hybrid methods are about 0.02 . It should be noted that $\overline{N F E}$ of the AS is almost three times those of the hybrid methods. The $\overline{N F E}$ values for both versions of the AWS are almost equal.

\subsection{Example 3: A conical structure under compressive axial load and bending moment}

In this example, the buckling reliability of a conical structure is studied. The perspective and different variables of this structure are demonstrated in Figure 11. As it can be seen, the structure is subjected to a compressive axial load $P$ and a bending moment $M$. The mechanical and geometrical variables are independent and follow a normal distribution as presented in Table 3 .

Table 3. Random variables for Example 3.

\begin{tabular}{|c|c|c|c|}
\hline \multicolumn{2}{|c|}{ Variable } & Mean & Deviation \\
\hline$X_{1}$ & $E(\mathrm{MPa})$ & 70000.00 & 3500.00 \\
\hline$X_{2}$ & $t(\mathrm{~m})$ & 0.0025 & 0.000125 \\
\hline$X_{3}$ & $\alpha(\mathrm{rad})$ & 0.524 & 0.010480 \\
\hline$X_{4}$ & $r_{1}(\mathrm{~m})$ & 0.90 & 0.022500 \\
\hline$X_{5}$ & $M(\mathrm{~N} . \mathrm{m})$ & 80000.00 & 6400.00 \\
\hline$X_{6}$ & $P(\mathrm{~N})$ & 70000.00 & 5600.00 \\
\hline
\end{tabular}




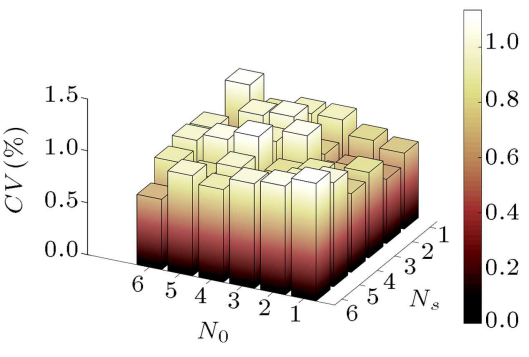

Sensitivity analysis of $C V$ for AWS-1

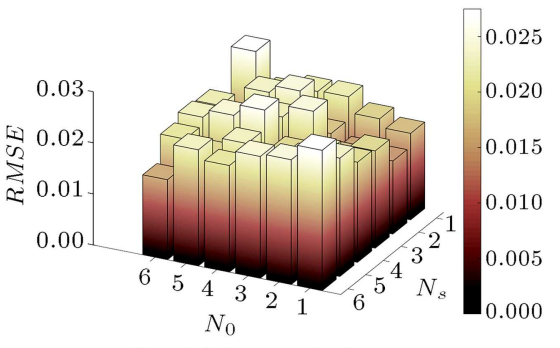

Sensitivity analysis of $R M S E$ for AWS-1

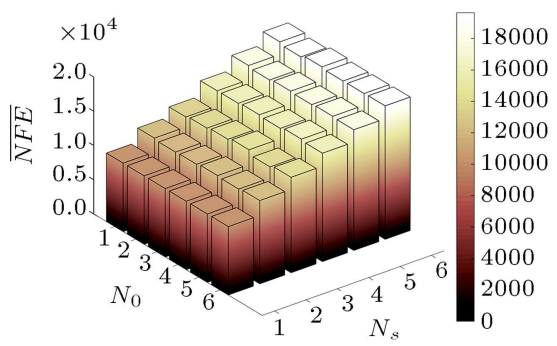

Sensitivity analysis of $\overline{N F E}$ for AWS-1

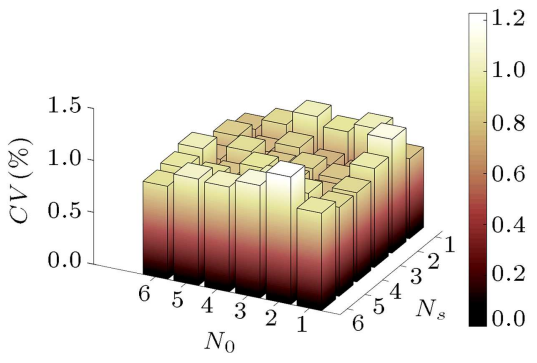

Sensitivity analysis of $C V$ for AWS-2

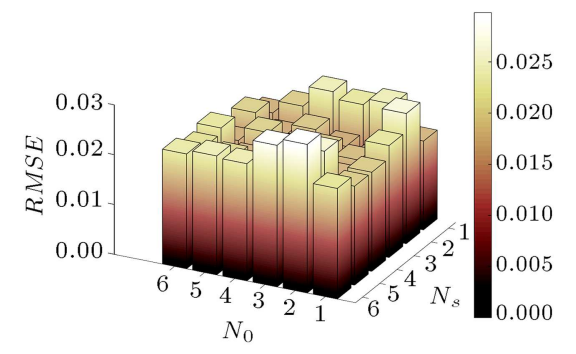

Sensitivity analysis of $R M S E$ for AWS-2

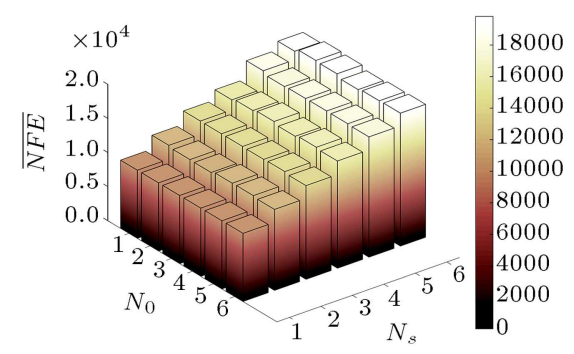

Sensitivity analysis of $\overline{N F E}$ for AWS-2

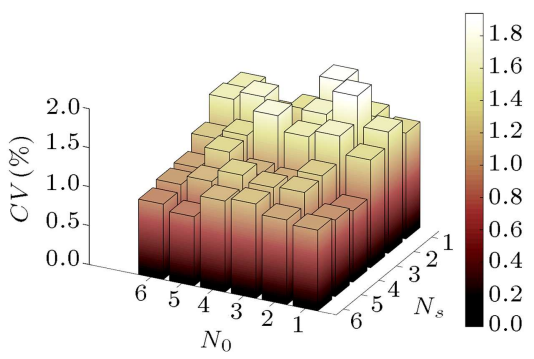

Sensitivity analysis of $C V$ for AS

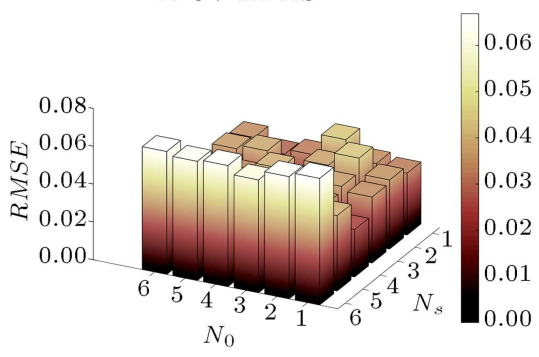

Sensitivity analysis of $R M S E$ for AS

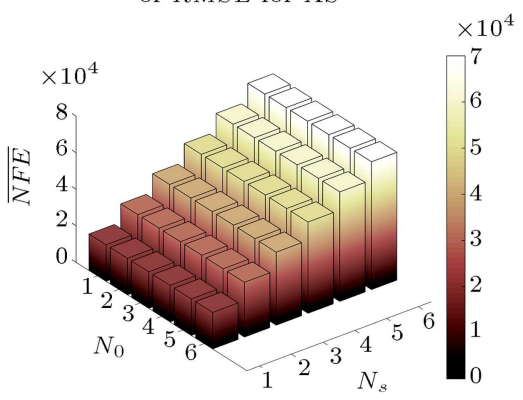

Sensitivity analysis of $\overline{N F E}$ for AS

Figure 10. Sensitivity analysis of $C V, R M S E$, and $\overline{N F E}$ for Example 2.

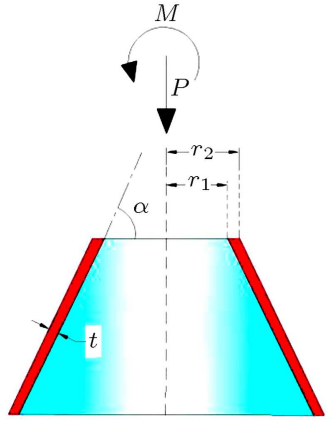

(a)

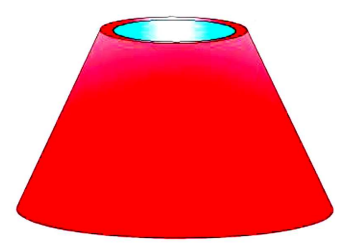

(b)
Figure 11. Schematics of the conical structure: (a) Random variables and (b) 3D perspective view.

The failure of this structure can happen by buckling due to instability or loss of strength. In the analysis, only the buckling mode will be investigated [23]. The buckling criterion is [24]:

$$
\frac{P}{P_{\text {crit. }}}+\frac{M}{M_{\text {crit. }}} \geq 1,
$$

where $P_{\text {crit. }}$ and $M_{\text {crit. }}$ are the critical axial load and bending momentum for buckling, respectively. They are calculated by the following equations:

$$
\begin{aligned}
& P_{\text {crit. }}=\gamma \frac{2 \pi E t^{2} \cos ^{2} \alpha}{\sqrt{3\left(1-\mu^{2}\right)}}, \\
& M_{\text {crit. }}=\eta \frac{\pi E t^{2} r_{1} \cos ^{2} \alpha}{\sqrt{3\left(1-\mu^{2}\right)}} .
\end{aligned}
$$

$\gamma$ and $\eta$ are respectively considered equal to 0.33 and 0.41 for correlation of the theoretical results with the experimental ones [24].

According to Eqs. (19)-(21), the limit state function can be formulated as:

$$
g(X)=1-\frac{\sqrt{3\left(1-\mu^{2}\right)}}{\pi X_{1} X_{2}^{2} \cos ^{2} X_{3}}\left(\frac{X_{6}}{2 \gamma}+\frac{X_{5}}{\eta X_{4}}\right) .
$$

The variables are defined in Table 3 . The failure probability of this engineering example is $1.86 \times 10^{-6}$ and the reliability index is $\beta=4.63$, which are obtained using the MCS with 100 million samples. 


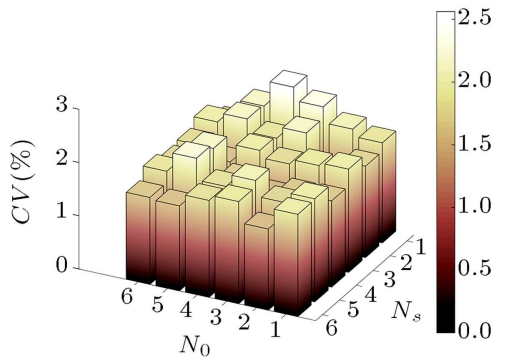

Sensitivity analysis of $C V$ for AWS-1

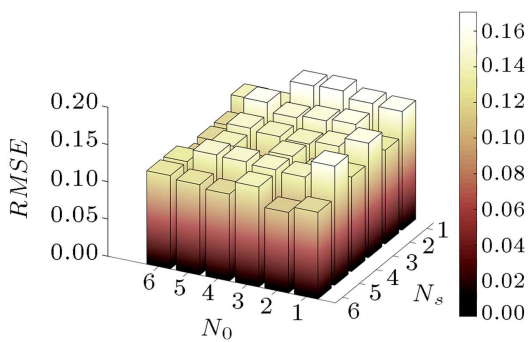

Sensitivity analysis of $R M S E$ for AWS-1

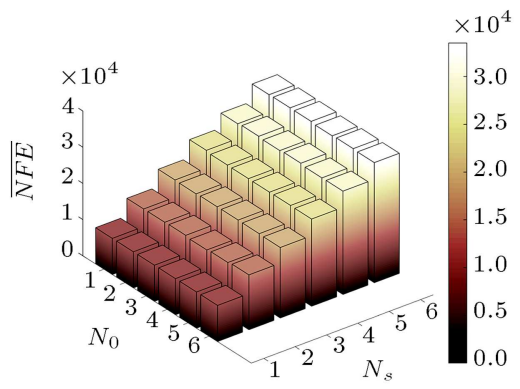

Sensitivity analysis of $\overline{N F E}$ for AWS-1

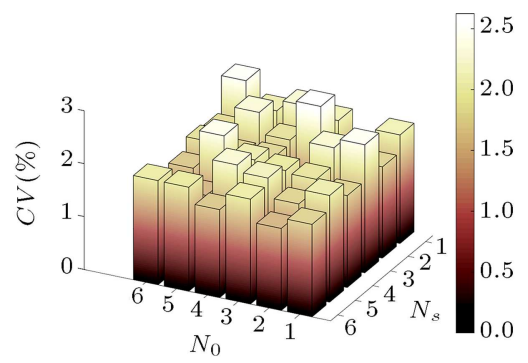

Sensitivity analysis of $C V$ for AWS-2

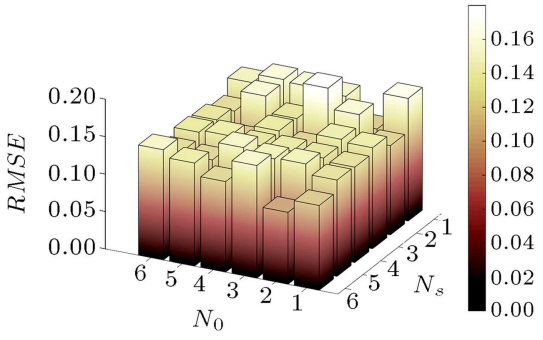

Sensitivity analysis of $R M S E$ for AWS-2

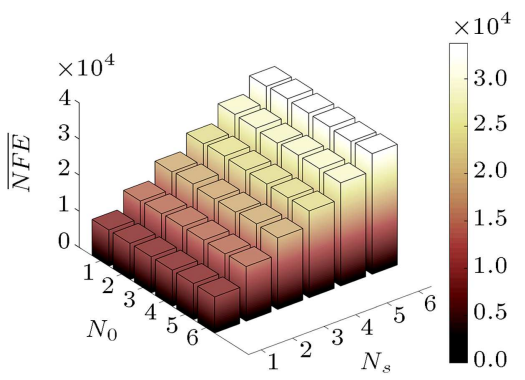

Sensitivity analysis of $\overline{N F E}$ for AWS-2

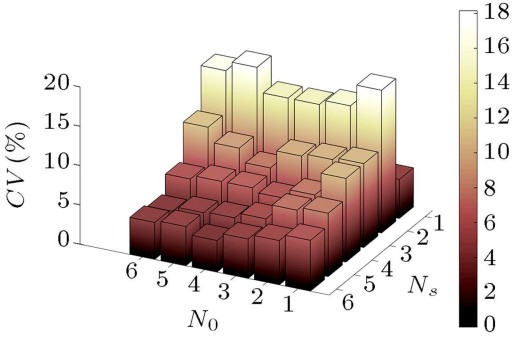

Sensitivity analysis of $C V$ for $\mathrm{AS}$
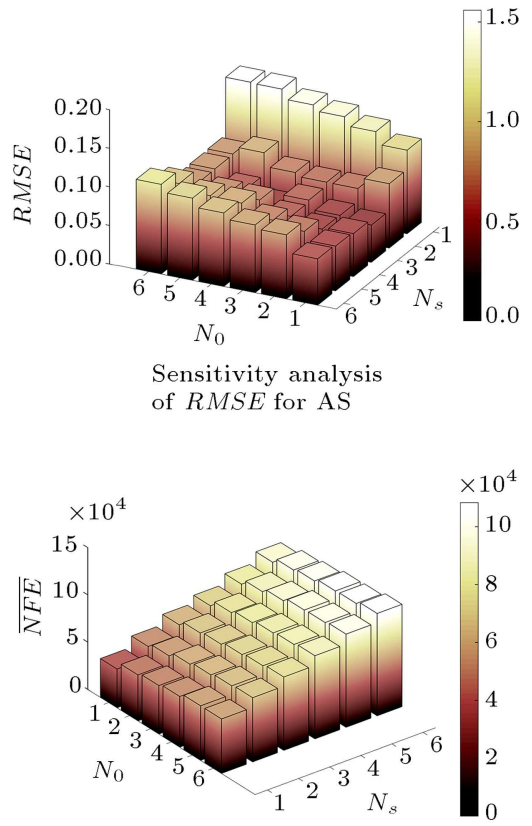

Sensitivity analysis of $\overline{N F E}$ for AS

Figure 12. Sensitivity analysis of $C V, R M S E$, and $\overline{N F E}$ for Example 3.

The results of different methods for approximately equal values of $\overline{N F E}$ are given in Table 4 . As can be seen, by considering $\overline{N F E}=1300$, unlike the asymptotic methods, MCS and WS cannot find any failed samples. However, the AWS methods can estimate the reliability index with RMSEs less than

Table 4. Comparison of the performances of the different methods for Example 3.

\begin{tabular}{cccccc}
\hline & MCS & AS & WS & AWS-1 & AWS-2 \\
\hline$\overline{N F E}$ & 1300 & 1274 & 1300 & 1271 & 1219 \\
$\bar{\beta}$ & - & 5.52 & - & 5.16 & 5.12 \\
$R M S E$ & - & 1.55 & - & 0.75 & 0.63 \\
$C V(\%)$ & - & 23.29 & - & 10.32 & 8.01 \\
\hline$\overline{N F E}$ & 12000 & 11880 & 12000 & 11062 & 11134 \\
$\bar{\beta}$ & - & 5.49 & 4.73 & 4.63 & 4.63 \\
$R M S E$ & - & 1.00 & 0.12 & 0.12 & 0.13 \\
$C V(\%)$ & - & 9.51 & 1.34 & 2.97 & 3.36 \\
\hline
\end{tabular}

0.8. By increasing $\overline{N F E}$ to 12000 , the WS estimates the failure probability with good accuracy, and the AWS-1 and 2 methods achieve averagely exact results.

By considering $N=10000$, the sensitivity of $C V$, $R M S E$, and $\overline{N F E}$ of the asymptotic methods to $N_{s}$ and $N_{0}$ is provided in Figure 12. The average values of $C V$ for AWS- 1 and 2 are about 1.9, while this value for $\mathrm{AS}$ is 8.2 and the minimum value of $\mathrm{AS}$ is equal to 3.4 , which is higher than the maximum $C V$ of the introduced methods. The maximum values of $R M S E$ for AWS-1 and 2 are 0.17 and 0.18 , respectively, which are less than the minimum AS $(R M S E=0.48)$. As expected, the value of $\overline{N F E}$ increases by increasing the number of support points. $\overline{N F E}$ of the AS is almost three to four times that of the proposed methods. In comparison of the two versions of the AWS, it can be seen that $\overline{N F E} s$ of the second version are slightly lower than those of the first version $(0.2 \%)$. 


\subsection{Example 4: The roof truss problem}

This example is a well-known problem studied in the literature $[25,26]$. The geometry details and loading of the roof truss are illustrated in Figure 13. As can be seen, the compression bars and top boom (red members) are reinforced, but the tension bars and the bottom boom (blue members) are made of steel. A uniformly distributed load $q$ is assigned to roof truss, which is transformed into nodal loads $p=q l / 4$. The perpendicular deflection of the peak node $(C)$ is limited to $3 \mathrm{~cm}$, so the limit state function can be constructed by Eq. (23):

$$
g(X)=0.03-\Delta_{C},
$$

where $\Delta_{C}$ can be obtained by:

$$
\Delta_{C}=\frac{q l^{2}}{2}\left(\frac{3.81}{A_{C} E_{C}}+\frac{1.13}{A_{S} E_{S}}\right),
$$

in which $l, A$, and $E$ are the length, area, and module of elasticity of the members, respectively, and $C$ and $S$ subscriptions are related to concrete and steel members, respectively. The details of random
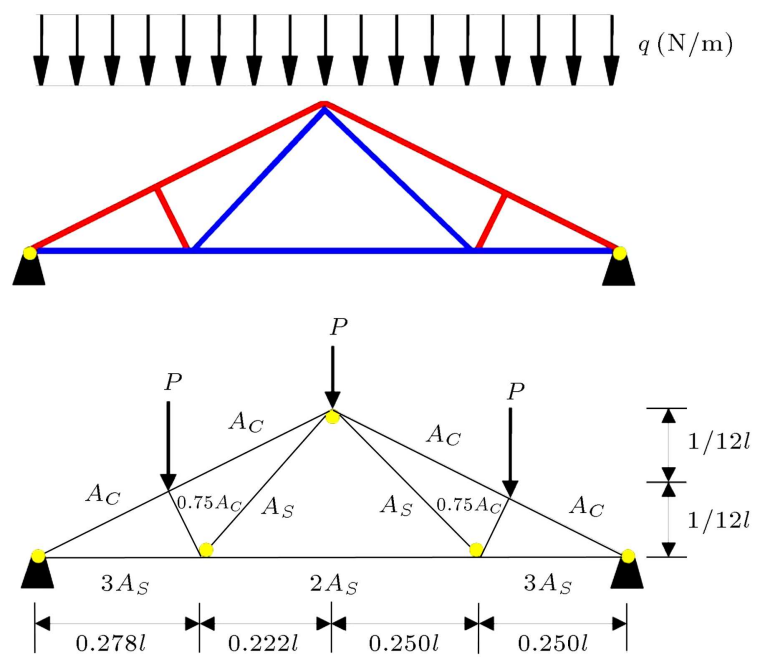

Figure 13. Roof truss: (a) Steel and concrete members and (b) geometry and loading. variables are provided in Table 5. Using MCS with 100 million samples, the failure probability is obtained as $P_{f}=0.00936$ and the reliability index is obtained as $\beta=2.35$.

The statistical results by considering three sets of experiments with $\overline{N F E}$ values almost equal to 110 , 300 , and 3000 are provided in Table 6 . In the first set, the asymptotic methods estimate the reliability index based on the experiments. Neither MCS nor WS can obtain the failed sample. The RMSE and CV of the proposed methods are also better than those of the AS. Increasing $\overline{N F E}$ to 300 evaluations enables the WS to estimate the reliability index. However, AS and WS are outperformed by AWS- 1 and 2 in terms of $R M S E$ and $C V$. In the third set, because of the higher number of samples for the low reliability index, the MCS outperforms other methods. However, the results of the other methods are also acceptable and AWS-1 is ranked the second for this set.

Since the failure probability of this example is relatively high, $N$ is considered 500 to perform the parameter study of the methods. According to Figure 14, the $C V$ and $R M S E$ of the AS method

Table 6. Comparison of the performances of the different methods for Example 4.

\begin{tabular}{cccccc}
\hline & MCS & AS & WS & AWS-1 & AWS-2 \\
\hline$\overline{N F E}$ & 110 & 116 & 110 & 104 & 106 \\
$\bar{\beta}$ & - & 2.08 & - & 2.72 & 2.67 \\
$R M S E$ & - & 2.05 & - & 0.59 & 0.54 \\
$C V(\%)$ & - & 99.21 & & 17.55 & 16.39 \\
\hline$\overline{N F E}$ & 300 & 300 & 300 & 300 & 300 \\
$\bar{\beta}$ & - & 2.30 & 2.42 & 2.51 & 2.47 \\
$R M S E$ & - & 0.47 & 0.29 & 0.29 & 0.25 \\
$C V(\%)$ & - & 20.70 & 11.63 & 9.86 & 9.18 \\
\hline$\overline{N F E}$ & 3000 & 3000 & 3000 & 3000 & 3000 \\
$\bar{\beta}$ & 2.36 & 2.40 & 2.38 & 2.34 & 2.37 \\
$R M S E$ & 0.06 & 0.13 & 0.12 & 0.10 & 0.12 \\
$C V(\%)$ & 2.65 & 5.32 & 4.91 & 4.35 & 5.1 \\
\hline
\end{tabular}

Table 5. Random variables for Example 4.

\begin{tabular}{llll}
\hline \multicolumn{1}{c}{ Variable } & Distribution type & \multicolumn{1}{c}{ Mean } & Standard deviation \\
\hline Uniform load, $q(\mathrm{~N} / \mathrm{m})$ & Normal & 20000 & 1400 \\
Length, $l(\mathrm{~m})$ & Normal & 12 & 0.12 \\
Sectional area, $A_{s}\left(\mathrm{~m}^{2}\right)$ & Normal & $9.82 \times 10^{-4}$ & $5.892 \times 10^{-5}$ \\
Sectional area, $A_{c}\left(\mathrm{~m}^{2}\right)$ & Normal & 0.04 & $4.8 \times 10^{-3}$ \\
Elastic modulus, $E_{s}\left(\mathrm{~N} / \mathrm{m}^{2}\right)$ & Normal & $1 \times 10^{11}$ & $6 \times 10^{9}$ \\
Elastic modulus, $E_{c}\left(\mathrm{~N} / \mathrm{m}^{2}\right)$ & Normal & $2 \times 10^{10}$ & $1.2 \times 10^{9}$ \\
\hline
\end{tabular}




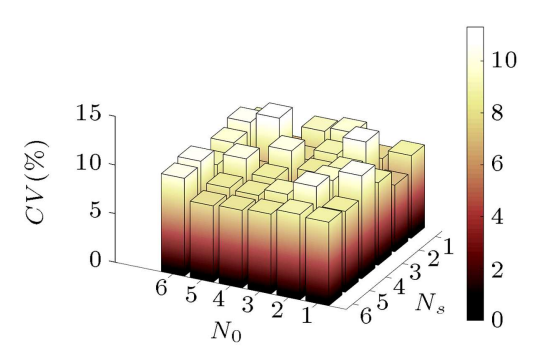

Sensitivity analysis of $C V$ for AWS-1

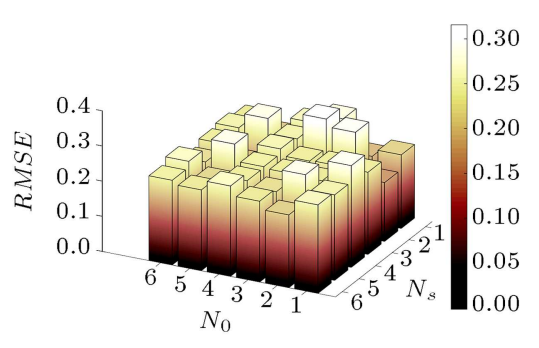

Sensitivity analysis of $R M S E$ for AWS-1

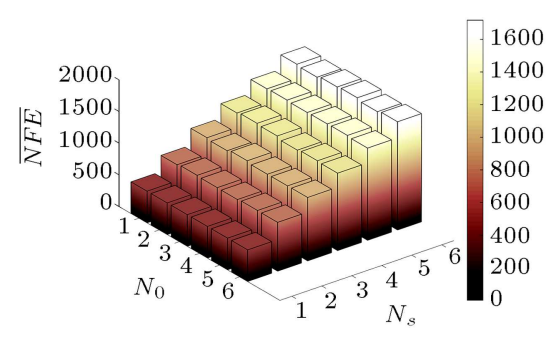

Sensitivity analysis of $\overline{N F E}$ for AWS-1
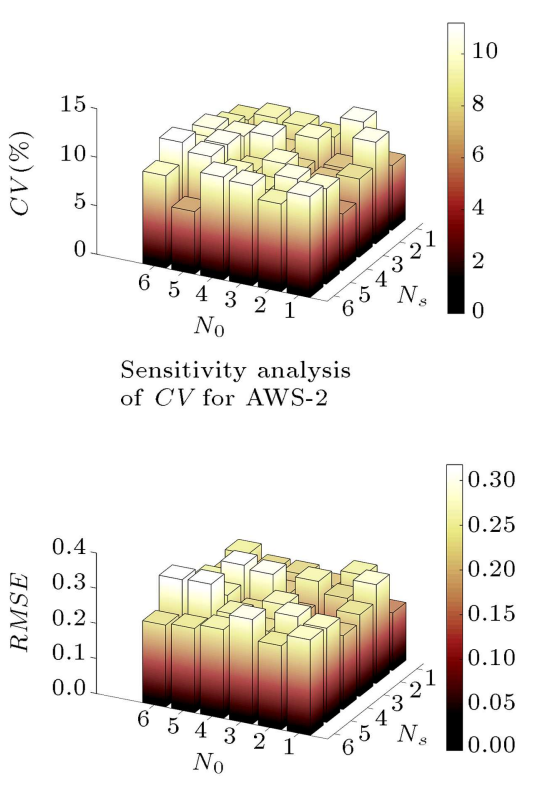

Sensitivity analysis of RMSE for AWS-2

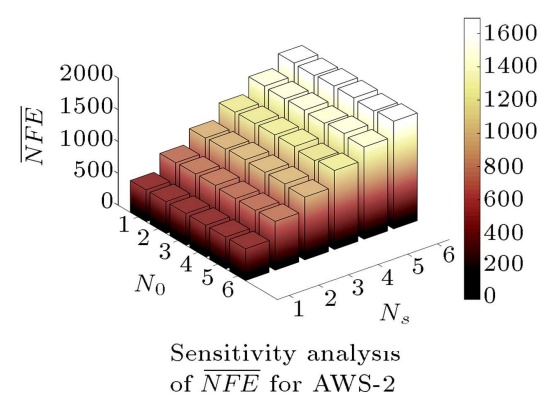

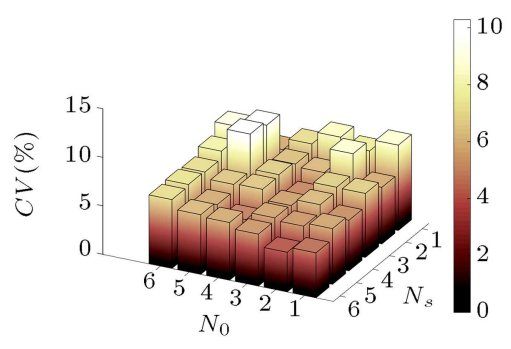

Sensitivity analysis of $C V$ for AS

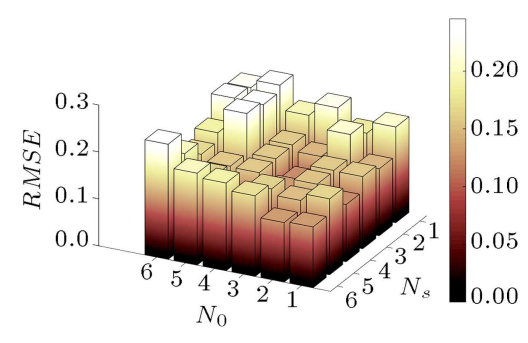

Sensitivity analysis of $R M S E$ for AS

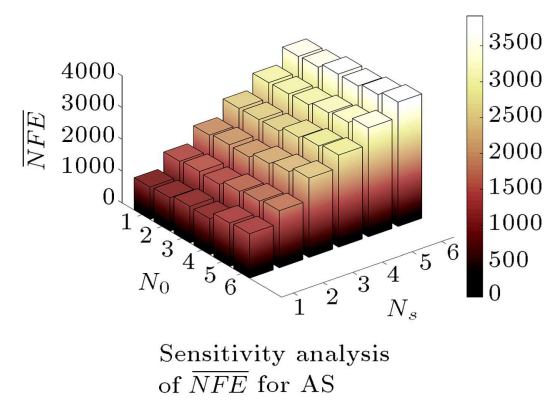

Figure 14. Sensitivity analysis of $C V, R M S E$, and $\overline{N F E}$ for Example 4.

are 0.17 and $6.5 \%$, while these values for AWS- 1 are 0.24 and $8.8 \%$, and for AWS-2 are 0.24 and $9.0 \%$, respectively. It should be noted that $\overline{N F E}$ values of the AWS-1 and AWS-2 are respectively 1113 and 1110, while for AS, it is 2419, which is almost two times that of the proposed methods.

\section{Concluding remarks}

This paper proposes a new hybrid reliability method for approximating the reliability index, in which the Weighted Simulation (WS) is accompanied by Asymptotic Sampling (AS). The WS provides a means of making samples of a specific standard deviation compatible with another standard deviation only by adjusting the weight indexes. On the other hand, AS is an efficient sampling method, which estimates the reliability index using the Monte Carlo Sampling (MCS) with differently scaled deviations. It was found that the AS method was a suitable approach to applying the abovementioned feature of the WS. This property made the AS an appropriate tool to find rare failure probabilities efficiently. Moreover, in comparison with the MCS, the WS estimated the failure probability by generating a low number of samples. The algorithm of the new method was designed and two versions were considered. In theory, the second version seemed to be more computationally economical; however, the first version was simpler than the second one. In order to verify the capabilities of the proposed method, several experiments were conducted. This study showed that the proposed method meaningfully reduced the number of state function evaluations (i.e., from two to five times, dependent on the problem and parameter setting) in comparison to the standard AS. Also, it outperformed the AS method in most cases. When the WS method was unable to find the failure samples, the hybrid method was capable to do so. The results also indicated the computational efficiency of the proposed method. It should be mentioned that the proposed method is very useful specifically when the failure probability is low, a large number of samples are needed (e.g., for MCS), and the evaluation of the limit state function is computationally expensive. In such cases, it can esti- 
mate the reliability index of the problem by using fewer evaluations and providing an acceptable accuracy.

\section{References}

1. Zio, E. "Reliability engineering: Old problems and new challenges", Reliability Engineering \& System Safety, 94(2), pp.125-141 (2009).

2. Selvik, J.T. and Signoret, J.-P. "How to interpret safety critical failures in risk and reliability assessments", Reliability Engineering \& System Safety, 161, pp. 61-68 (2017).

3. Thoft-Cristensen, P. and Baker, M.J., Structural Reliability Theory and Its Applications, Springer Science \& Business Media (2012).

4. Frangopol, D.M. and Maute, K. "Life-cycle reliabilitybased optimization of civil and aerospace structures", Computers \& Structures, 81(7), pp. 397-410 (2003).

5. Chakraborty, S. and Majumder, D. "Hybrid reliability analysis framework for reliability analysis of tunnels", Journal of Computing in Civil Engineering, 32(4), p. 04018018 (2018).

6. Okasha, N.M. "Proposed algorithms for an efficient system reliability-based design optimization of truss structures", Journal of Computing in Civil Engineering, 30(5), p. 04016008 (2016).

7. Bucher, C., Computational Analysis of Randomness in Structural Mechanics, CRC Press, London (2009).

8. Hasofer, A.M. and Lind, N.C. "Exact and invariant second-moment code format", Journal of the Engineering Mechanics Division, 100(1), pp. 111-121 (1974).

9. Aslam, M., Tahir, M., and Hussain, Z. "Reliability analysis of 3-component mixture of distributions", Scientia Iranica, 25(3), pp. 1768-1781 (2018).

10. Macke, M. and Bucher, C. "Importance sampling for randomly excited dynamical systems", Journal of Sound and Vibration, 268(2), pp. 269-290 (2003).

11. Bucher, C. "Asymptotic sampling for high-dimensional reliability analysis", Probabilistic Engineering Mechanics, 24(4), pp. 504-510 (2009).

12. Rashki, M., Miri, M., and Moghaddam, M.A. "A new efficient simulation method to approximate the probability of failure and most probable point", Structural Safety, 39, pp. 22-29 (2012).

13. Kaveh, A., Advances in Metaheuristic Algorithms for Optimal Design of Structures, Springer, Switzerland (2017).

14. Kaveh, A. and Dadras, A. "A novel meta-heuristic optimization algorithm: Thermal exchange optimization", Advances in Engineering Software, 110, pp. 6984 (2017).
15. Kaveh, A., Dadras, A., and Montazeran, A.H. "Chaotic enhanced colliding bodies algorithms for size optimization of truss structures", Acta Mechanica, 229(7), pp. 2883-2907 (2018).

16. Kaveh, A., Dadras, A., and Malek, N.G. "Buckling load of laminated composite plates using three variants of the biogeography-based optimization algorithm", Acta Mechanica, 229(4), pp. 1551-1566 (2018).

17. A. Rahmati, S.H., Ahmadi, A., and Karimi, B. "Developing simulation based optimization mechanism for a novel stochastic reliability centered maintenance problem", Scientia Iranica, 25(5), pp. 2788-2806 (2018).

18. Gharib, Z., Bozorgi-Amiri, A., Tavakkoli-Moghaddam, R., and Najafi, E. "A cluster-based emergency vehicle routing problem in disaster with reliability", Scientia Iranica, 25(4), pp. 2312-2330 (2018).

19. Breitung, K. "Asymptotic approximations for multinormal integrals", Journal of Engineering Mechanics, 110(3), pp. 357-366 (1984).

20. Gasser, C. and Bucher, C. "An optimized strategy for using asymptotic sampling for reliability analysis", Structural Safety, 71, pp. 33-40 (2018).

21. Chen, X. and Li, J. "A subset multicanonical Monte Carlo method for simulating rare failure events", Journal of Computational Physics, 344, pp. 23-35 (2017).

22. Kim, S.-H. and Na, S.-W. "Response surface method using vector projected sampling points", Structural Safety, 19(1), pp. 3-19 (1997).

23. Elegbede, C. "Structural reliability assessment based on particles swarm optimization", Structural Safety, 27(2), pp. 171-186 (2005).

24. Weingarten, V. and Seide, P. "NASA SP-8019buckling of thin-walled truncated cones", NASA Space vehicle Design Criteria-Structures (1968).

25. Rashki, M., Miri, M., and Moghaddam, M.A. "A simulation-based method for reliability based design optimization problems with highly nonlinear constraints", Automation in Construction, 47, pp. 24-36 (2014).

26. Song, S., Lu, Z., and Qiao, H. "Subset simulation for structural reliability sensitivity analysis", Reliability Engineering \& System Safety, 94(2), pp. 658-665 (2009).

\section{Biographies}

Ali Kaveh was born in 1948 in Tabriz, Iran. After graduation from the Department of Civil Engineering at the University of Tabriz in 1969, he continued his studies on Structures at Imperial College of Science and Technology at London University, and received his MSc, DIC and PhD degrees in 1970 and 1974, respectively. He then joined the Iran University of Science and Technology. Professor Kaveh is the author of 620 papers published in international journals and 
155 papers presented at national and international conferences. He has authored 23 books in Persian and 10 books in English published by Wiley, Research Studies Press, American Mechanical Society and Springer.

Armin Dadras Eslamlou was born in Urmia, Iran, in 1993. He is currently a $\mathrm{PhD}$ candidate in the School of Civil Engineering at Iran University of Science and Technology under the supervision of Prof.
A. Kaveh. He is interested in the development, enhancement, and application of statistical and soft computing methods to engineering problems. He has published over 10 journal papers and conference articles on structural design and health monitoring. He has repeatedly been appreciated as an outstanding researcher and supported by Ministry of Science Research and Technology and Iran's National Elites Foundation. 